\title{
Recent advances on polaprezinc for medical use (Review)
}

\author{
MINGRU LI ${ }^{1 *}$, ZHEN SUN $^{2 *}$, HONG ZHANG ${ }^{1}$ and ZHAOYANG LIU ${ }^{3}$ \\ ${ }^{1}$ Jilin Broadwell Pharmaceutical Co., Ltd., Liaoyuan, Jilin 136200; \\ ${ }^{2}$ Department of Gastroenterology, Jilin People's Hospital, Jilin City, Jilin 132000; \\ ${ }^{3}$ National Cancer Center/National Clinical Research Center for Cancer/Cancer Hospital, \\ Chinese Academy of Medical Sciences and Peking Union Medical College, Beijing 100021, P.R. China
}

Received February 25, 2021; Accepted July 6, 2021

DOI: $10.3892 / \mathrm{etm} .2021 .10880$

\begin{abstract}
The present study described the chemical and biological properties of zinc complex of L-carnosine (L-CAZ; generic name, polaprezinc; chemical name, catena-(S)- $[\mu-[\mathrm{N}($

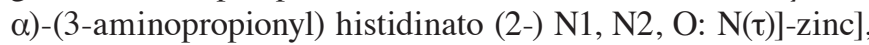
molecular formula, $\mathrm{C}_{9} \mathrm{H}_{14} \mathrm{~N}_{4} \mathrm{O}_{3} \mathrm{Zn}$; molecular weight, 291.6404; CAS registry number, 107667-60-7). Characterized as a white or yellowish white crystalline powder, this drug is insoluble in glacial acetic acid and almost insoluble in water, methanol, ethanol and ether. It is soluble in dilute hydrochloric acid, dilute nitric acid and sodium hydroxide solution, and its melting point is $260-270^{\circ} \mathrm{C}$. Polaprezinc is an anti-ulcer drug that was jointly studied and developed by Hamari Chemicals Co., Ltd. and Zeria Pharmaceutical Co., Ltd., and was first approved in Japan in 1994. This review article summarizes the research advances of polaprezinc, including the patents, preparations, synthetic routes, pharmacokinetics, pharmacological effects and application in clinical research.
\end{abstract}

\section{Contents}

1. Introduction

2. Patents and preparations

3. Synthetic route and pharmacokinetics of polaprezinc

4. Pharmacological study of polaprezinc

5. Clinical uses of polaprezinc

6. Other clinical applications

7. Safety

8. Conclusion

Correspondence to: Dr Zhaoyang Liu, National Cancer Center/National Clinical Research Center for Cancer/Cancer Hospital, Chinese Academy of Medical Sciences and Peking Union Medical College, 17 Pan Jia Yuan Nan Li, Chaoyang, Beijing 100021, P.R. China E-mail: liuzhy118@163.com

*Contributed equally

Key words: polaprezinc, membrane protection, Helicobacter pylori, antioxidant, anti-apoptosis

\section{Introduction}

Polaprezinc, a chelated form of zinc ( $\mathrm{Zn})$ and L-carnosine, is a new generation gastric mucosal protective agent that has been used in clinical for more than 20 years in Japan (1). The structural formula is presented in Fig. 1. Polaprezinc can improve the eradication rates of Helicobacter pylori, which has been verified in clinical practice (2). Polaprezinc also provides mucosal protection by increasing heat shock protein expression (3-5) and presenting antioxidant (6) and antiapoptotic (7) effects. It can also inhibit the expression of inflammatory factors (8-10), as well as stimulate the proliferation and migration of granulation tissue in injured epithelial cells (11). Polaprezinc can therefore promote the healing of peptic ulcers and improve the quality of ulcer healing $(12,13)$. Polaprezinc might have a broad clinical application prospect.

\section{Patents and preparations}

Patents. Japan applied to the first world patent for polaprezinc in 1989 (patent no. WO9015616), which is an application patent for gastric ulcer, followed by a patent application for liver fibrosis in 2004 (patent no. JP4802470B2) (14) and a patent application for an orally disintegrating tablet in 2016 (patent no. JP2017002045A) (15). However, the patent application for the core compound of polaprezinc was not covered in China. In 2012, China produced its first generic drug indicated for gastric ulcer, which was approved for listing by the State Food and Drug Administration (Jilin Broadwell Pharmaceutical Co., Ltd.). In 2016, a related patent was applied for in China. The subjects of the application and authorization were relatively similar, in terms of compounds, preparation methods and application patents (16-18).

Preparations. Polaprezinc preparations in Japan include granules, tablets and orally disintegrating tablets, whereas those in China mainly come in the form of granules. To improve the dissolution of polaprezinc, the granular formulation contains polaprezinc, ethanol, mannitol, povidone K-30, cyclodextrin, trehalose, maltitol and pregelatinized starch prepared dispersion. This formula can also enhance the stability and bioavailability of polaprezinc. The combination preparation of polaprezinc and other active ingredients also represents an effective research and development strategy. An in vitro 
pharmacological study demonstrated that, combined with the conventional antibiotics levofloxacin, doxycycline, ceftazidime and clarithromycin, polaprezinc exhibits an antibacterial activity superior to that of the antibiotic-alone group, indicating a significant synergistic effect (19). Furthermore, the combination of polaprezinc and proton pump inhibitors (PPIs) can significantly accelerate the rate of ulcer healing following endoscopic submucosal dissection (ESD), leading to improved quality of healing and reduced incidence of postoperative complications (20).

\section{Synthetic route and pharmacokinetics of polaprezinc}

Synthesis route. Polaprezinc is a complex of L-carnosine and $\mathrm{Zn}$. Thus, the synthesis of polaprezinc can be decomposed into L-carnosine synthesis and L-carnosine-Zn salt complexation. b-Dihydro-1,3-thiazol-2,4-dione was synthesized from alanine and methyl o-ethyl xanthate, then acylated with L-histidine to obtain L-carnosine and finally complexed with zinc acetate to obtain polaprezinc.

Pharmacokinetics. The intestinal absorption of L-CAZ was studied in rats by Matsukura and Tanaka (21) using ${ }^{14} \mathrm{C}$ - and ${ }^{65} \mathrm{Zn}$-labeled compounds. L-CAZ was suggested to dissociate to its components, L-carnosine and $\mathrm{Zn}$, during intestinal absorption. After a single administration of ${ }^{14} \mathrm{C}$-labeled L-CAZ to rats, the accumulated excretion rates of L-CAZ were $4.1 \%$ in urine, $13.3 \%$ in feces, and $38.8 \%$ in exhalation. For ${ }^{65} \mathrm{Zn}$-labeled L-CAZ, the excretion rates were $0.3 \%$ in urine and $85.0 \%$ in feces. The absorption rate of $\mathrm{Zn}$ was estimated to be $11 \%$.

In another study, seven healthy subjects received polaprezinc $(75 \mathrm{mg}$ ) in a single oral administration (22). The highest plasma $\mathrm{Zn}$ concentration was reached $1.6 \mathrm{~h}$ after the drug was ingested and was $1.9 \mu \mathrm{g} / \mathrm{ml}$.

Evaluation of the rat model of acetic acid-induced ulcer showed that the $\mathrm{Zn}$ concentration at the ulcer site was still higher compared with that before administration and $12 \mathrm{~h}$ after polaprezinc $(50 \mathrm{mg} / \mathrm{kg}$ ) was administered. During absorption, the product dissociates into carnosine, which is metabolized into L-histidine and aminoacrylic acid. The amino acids and absorbed $\mathrm{Zn}$ are then metabolized according to the endogenous metabolic system (23). $\mathrm{Zn}$ is mainly excreted in the feces, with excretion rates of $0.47 \%$ when taking polaprezinc $(150 \mathrm{mg})$ on an empty stomach and of $0.12 \%$ after a meal. If polaprezinc is taken orally for 7 consecutive days, the excretion rate of $\mathrm{Zn}$ in urine for one day is $0.21-0.46 \%$. If $300 \mathrm{mg}$ of polaprezinc is taken on an empty stomach, the excretion rates of $\mathrm{Zn}$ in feces in 24 and $48 \mathrm{~h}$ are 41.4 and $58.8 \%$, respectively. The $\mathrm{Zn}$ content in feces is twice that before taking the drug because the accumulation rate of $\mathrm{Zn}$ is relatively low.

\section{Pharmacological study of polaprezinc}

Anti-ulcer effect. Polaprezinc is effective in all experimental models, including emergency gastric injury, aspirin hydrochloride gastric injury, histamine gastric injury and ethanol hydrochloric acid gastric injury (24), and presents a dose-dependent efficiency, particularly for ethanol hydrochloride gastric injury and duodenal ulcer. An in vitro experiment demonstrated that polaprezinc can inhibit the secretion of pepsin in rat gastric epithelial cell line RGM1 and therefore significantly reduce the damage to gastric mucosa. Polaprezinc is therefore an anti-ulcer drug with enhanced defensive factors (25).

Anti-Helicobacter pylori (H. pylori) effect. Previous in vivo and in vitro studies have confirmed that polaprezinc exerts an anti-H. pylori effect (19), which is mainly attributed to $\mathrm{Zn}$ ions. Polaprezinc can significantly increase antibacterial activity in the stomach due to its chelating structure and because it can effectively adhere to damaged gastric mucosa. The results indicated that the eradication rate of $H$. pylori in polaprezinc $(150 \mathrm{mg} / \mathrm{d})$ combined with conventional triple therapy (omeprazole $40 \mathrm{mg} / \mathrm{d}$, amoxicillin $2 \mathrm{~g} / \mathrm{d}$ and clarithromycin $1 \mathrm{~g} / \mathrm{d})$ was significantly higher compared with that of conventional triple therapy (ITT 77 vs. 58.6\%, PP 81.1 vs. 61.4\%) (2). $H$. pylori produces a large amount of active urease, which is an important pathogenic factor for H. pylori infection. $\mathrm{Zn}$ and L-carnosine, two important components of polaprezinc, can significantly inhibit urease activity. Urease is a nickel metalloenzyme, which activity is determined by the nickel content of the active site. The urease gene cluster ureABIEFGH contains at least seven essential genes, including ure $\mathrm{A}$ and ureB, which are structural genes, ureEFGH, which is an auxiliary gene and ureI, which is a gene unique to $H p$ urease. Urease helper genes facilitate the insertion of nickel into ureA/ureB protein active sites to form active proteins. Urease activation requires the insertion of two nickels in the six active sites of the whole enzyme. The protein encoded by the helper gene plays therefore an important role in the polymerization of nickel ions to the active site of the enzyme. $\mathrm{Zn}$ can induce the substitution reaction of nickel ions in the urease molecule and change the conformation of the urease, significantly inhibiting its enzyme activity (26). In addition, carnosine is composed of alanine and histidine, and histidine can effectively adjust the $\mathrm{Zn}$ ion and accurately insert it into the structural site of the enzyme, significantly inhibiting urease activity. Carnosine can also regulate the accurate insertion of $\mathrm{Zn}$ ions into the active site of the zymogen and replace the nickel ion (27).

Promotion of healing. Polaprezinc promotes healing in the rat model of chronic ulcer (28). A study selected numerous acetate ulcer models (AAUs) and iron-ascorbic acid models (FAUs) to determine the ulcer area, and hydroxyproline (HYP) and DNA contents at the ulcer site were used as indicators (29). Following oral administration of polaprezinc at the concentrations of 1,3 and $10 \mathrm{mg} / \mathrm{kg}$, the ulcer areas of the models of AAUs and FAUs decreased on day 4 of ulceration, but the ulcer did not heal completely on Day 14 . The lysine content of the polaprezinc group increased on Days $4(4.3 \pm 0.8 \mu \mathrm{g} / \mathrm{mg})$ and 7 $(10.7 \pm 1.2 \mu \mathrm{g} / \mathrm{mg})$ of ulceration compared with the control group, and significantly increased on day 11 . The DNA content of the FAU model was significantly lower than that of the non-ulcer site on Day 7 of ulceration. However, the DNA content of the AAU model was not reduced, although the DNA content of the two model ulcer sites on Day 11 was significantly higher than that of the non-ulcer site. The contents returned to their normal levels after 14 days. For both models, the HYP content in the ulcer site was increased in a dose-dependent manner 


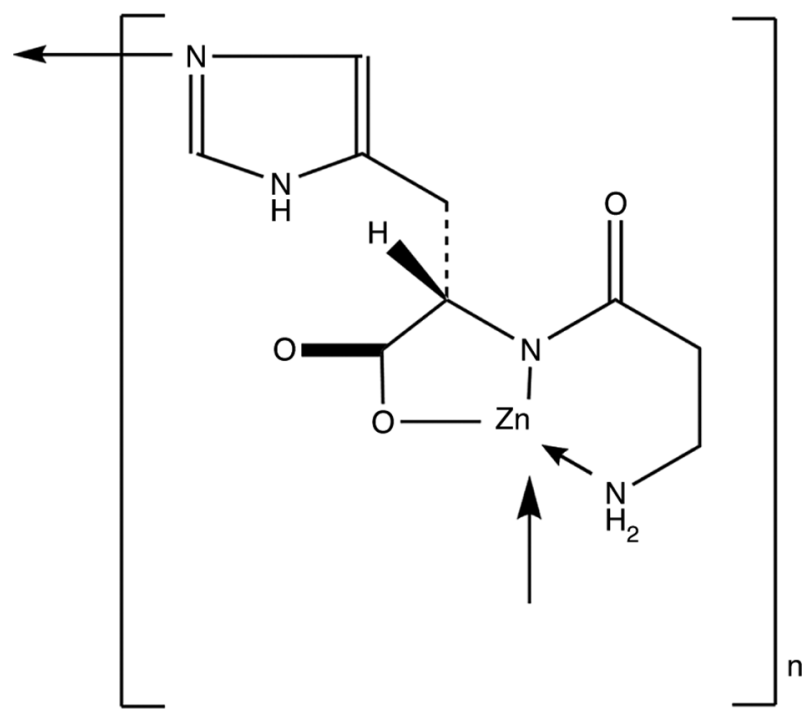

Figure 1. Structural formula of polaprezinc.

when a polaprezinc dose of 1-10 $\mathrm{mg} / \mathrm{g}$ was given (29). When a polaprezinc dose of $3 \mathrm{mg} / \mathrm{kg}$ was administered to the AAUs on Day 4 of ulceration and to FAUs on Day 7 of ulceration, the DNA contents returned to normal levels faster, compared with the control group (30). Furthermore, the healing effect of polaprezinc on ulcers is attributed to its antioxidative property. A previous study reported that polaprezinc significantly reduces the gastric ulcer area in a dose-dependent manner, with a concomitant reduction in the activities of xanthine oxidase and myeloperoxidase; meanwhile, it also reduces the malondialdehyde level in the ulcerated mucosa. Polaprezinc can restore the glutathione level in the mucosa. It can also consistently downregulate the protein expression of tumor necrosis factor- $\alpha$, interleukin- $1 \beta$, macrophage inflammatory protein- 2 and cytokine-induced neutrophil chemoattractant- $2 \mathrm{a}$, which are all activated in the ulcerated tissues to promote the healing of ulcer mucosa (31).

Anti-liver fibrosis. Hepatic fibrosis is caused by fibrocytes stimulated by certain infections, such as hepatitis B and hepatitis $\mathrm{C}$ infections, producing therefore a large amount of extracellular matrix (ECM). Up to $90 \%$ of patients with liver cancer present with advanced liver fibrosis or cirrhosis. Static hepatic stellate cells in the space around the liver sinusoids are the main producers of liver ECM and are considered as key factors for liver fibrosis (14,32). The study by Ye et al (33) reported that polaprezinc can inhibit the proliferation and migration of hepatic stellate cells. Furthermore, the expression of liver fibrosis markers, including type I collagen, fibronectin, and $\alpha$-smooth muscle actin, is downregulated following treatment with polaprezinc. Gene chip analysis through microarray analysis was performed using OneArray, which contains 31,741 mRNA probes, detecting 20,672 genes in the human genome. It was found that 202 differentially expressed genes were altered by $>1.5$ times after polaprezinc treatment, Changes in these genes were associated with tumor cell proliferation, migration and cytoskeletal structure. In the animal model of hepatic fibrosis induced by thioacetamide, $200 \mathrm{mg} /$ day polaprezinc significantly inhibits the expression of matrix metalloproteinase (MMP)-2 and MMP-9 while reducing the area of liver fibrosis. A Japanese clinical study reported that patients with hepatitis $\mathrm{C}$ and cirrhosis that were treated with $150 \mathrm{mg} /$ day polaprezinc for 3 years, had significantly decreased levels of alanine aminotransferase and aspartate aminotransferase after those 3 years compared with patients in the control group $(\mathrm{P}=0.0293)$. Taken together, these findings suggested that polaprezinc can be considered as a novel treatment method for patients with liver cirrhosis (34).

\section{Clinical uses of polaprezinc}

H. pylori eradication. A previous study reported that polaprezinc can significantly reduce urease activity and inhibit the levels of inflammation and oxidative stress caused by $H$. pylori infection (35). Kashimura et al (19) demonstrated that polaprezinc has a potential role in $H$. pylori eradication therapy. This study included 66 patients with $H$. pylori infection, and dyspepsia was studied for 7 days. The quadruple therapy group of patients (lansoprazole, amoxycillin, clarithromycin and polaprezinc) who received polaprezinc had a $17 \%$ higher $H$. pylori eradication rate than patients in the triple therapy group (lansoprazole, amoxycillin and clarithromycin) $(\mathrm{P}<0.05)$. To further compare the efficacy and safety of polaprezinc-containing quadruple therapy and triple therapy for the eradication of $H$. pylori, a prospective, multicenter, randomized, parallel, open-label, controlled and multicenter clinical study was conducted by the Department of Gastroenterology of Peking Union Medical College Hospital in 11 cities across the country. This study found a significant increase in the H.pylori eradication rate in the polaprezinc quadruple therapy compared with that in the standard triple therapy. The difference in rate reached $19.7 \%$ (per protocol analysis, 81.1 vs. 61.4\%) (2), suggesting that polaprezinc can also enhance resistance of the gastric mucosa to $H$. pylori infection and that polaprezinc could be used for $H$. pylori eradication treatment.

Application after ESD. With the introduction of ESD in the late 1990s, the indications for early gastric cancer endoscopic treatment have further expanded (36). Minimally invasive and exhibiting good efficacy, ESD is currently the standard method for endoscopic treatment of early gastric cancer and precancerous lesions (20). At present, patients following upper gastrointestinal ESD routinely use PPIs to stimulate ulcer healing. A meta-analysis conducted by Nishizawa et al (20) reported that PPI combined with gastric mucosal protective agents is significantly more effective than PPI alone. Furthermore, a study by Inaba et al (37) included 163 patients after ESD surgery, who were randomly assigned to take either lansoprazole daily $(30 \mathrm{mg})$ or lansoprazole $(30 \mathrm{mg})$ combined with polaprezinc $(150 \mathrm{mg})$ daily. After 2 months, the ulcer healing scores of patients who received the lansoprazole combined with polaprezinc treatment were significantly increased $(\mathrm{P}<0.0001)$ compared with the lansoprazole group, while the ulcer base protrusion rate was significantly decreased $(\mathrm{P}<0.0001)$. Polaprezinc therefore provides more choices for postoperative ESD drug applications.

Drug-related gastrointestinal mucosal injury. In a randomized controlled study $(n=20)$ conducted by Watari et al $(38)$, patients 
with small-bowel mucosal injuries that had been treated with low-dose aspirin for an extended time were orally administered polaprezinc $(150 \mathrm{mg})$ daily. After 4 weeks, the number of lower lesions (including erythema and ulcers) was significantly reduced in the polaprezinc treatment group compared with the control group, as revealed by capsule endoscopy $(\mathrm{P}<0.05)$. These results indicate that polaprezinc effectively protects the mucosa against low-dose aspirin-induced small-bowel injuries. The underlying mechanisms remain unknown and require further investigation.

\section{Mucosal damage caused by radiotherapy}

Oral mucositis. A prospective Japanese study reported that patients with head and neck tumors who received radiotherapy and chemotherapy combined with polaprezinc treatment had a lower incidence of severe oral mucositis compared with the control group who did not received polaprezinc (39.3 vs. 60.7\%) (39). In patients with leukemia who received chemotherapy and radiotherapy and an allogeneic bone marrow stem cell transplant (40), the incidence of moderate to severe oral mucositis is significantly reduced in the polaprezinc-treated group compared with the control group (20 vs. 82\%; $\mathrm{P}<0.01$ ). Ishihama et al (41) reported that a polaprezinc mouth rinse effectively improves oral mucosal injury in 423 patients with symptoms of oral mucosal injury as a result of cancer treatment. The effects of the polaprezinc rinse were evaluated according to the cancer treatment method. The stomatitis prevention success rate, symptom improvement rate, pain prevention success rate and symptom improvement rate were $68.5,84.4,75.4$ and $76.7 \%$, respectively, for patients who received chemotherapy $(\mathrm{n}=280), 32.7,64.5,45.5$ and $73.5 \%$, respectively, for patients who received chemoradiation therapy $(n=95)$, and 29.6, 60.0, 40.7 and $68.6 \%$, respectively, for patients who received radiotherapy alone.

Radioactive esophagitis. Hayashi et al (42) conducted a randomized controlled study on patients with non-small cell lung cancer treated with radiotherapy and chemotherapy at Gifu University Hospital between 2011 and 2015. The results demonstrated that the polaprezinc group can prevent patients from developing grade 2 or higher radiation esophagitis compared with the group not treated with polaprezinc (hazard ratio $=0.397 ; 95 \%$ confidence interval $=0.160-0.990 ; \mathrm{P}=0.047)$.

Radioactive gastroenteritis. Patients with hematological malignancies often require systemic radiotherapy before stem cell transplantation. One of the common adverse reactions is radiation gastritis. Masayuki et al (43) reported that oral administration of polaprezinc before oral systemic radiotherapy leads to a lower incidence of radiation gastritis $(\mathrm{P}=0.046)$. Furthermore, in another study, female Wister rats aged 5 weeks were treated with polaprezinc enema for 10 days following rectal radiation. Endoscopic and histological analyses demonstrated that mucosal inflammation is significantly reduced in theses rats compared with control group (44). Another study also reported that administration of $100 \mathrm{mg} / \mathrm{kg}$ polaprezinc $2 \mathrm{~h}$ before experiment can reduce the apoptosis of intestinal epithelial cells in the duodenum, jejunum and ileum during ion beam injury, protecting the normal epithelial cells in the intestine (45).
Treatment of ulcerative colitis. The pathogenesis of ulcerative colitis is currently unclear. It is a chronic, recurrent intestinal inflammatory disease characterized by diffuse inflammation and ulcers. A previous study demonstrated that serum zinc concentration is reduced in patients with inflammatory bowel disease (IBD), and that severe IBD patients have serum zinc deficiency. After the polaprezinc treatment, the serum zinc level can be increased and maintained in the normal range (46). A study of 2,4,6-trinitrobenzenesulphonic acid-induced colitis in rats, demonstrated that, compared with the control group, the polaprezinc $(60 \mathrm{mg} / \mathrm{kg} / \mathrm{d})$ group could significantly inhibit the activation of calcineurin and the expression of proinflammatory cytokines in the mucosa, thus improving the symptoms of ulcerative colitis (47). Moreover, an in vitro study demonstrated that the viability of $\mathrm{T}$ cells was not affected by polaprezinc (47).

\section{Other clinical applications}

Taste disorders. Taste disorders are often associated with oral mucositis and can be additional side effects of chemotherapy and radiotherapy (48). Cancer patients undergoing chemotherapy often experience changes in taste perception. Multiple evidence suggests that taste disorders are associated with zinc deficiency, since enzymes that require zinc are found in the taste buds and that they play an important role in the function of taste (49). A study was performed to evaluate the presence of taste alteration and the effectiveness of polaprezinc administration in 136 female patients with breast cancer who underwent FE100 therapy. Of 58 patients with taste alteration, 20 received polaprezinc, with the following outcomes: Taste alteration improved in $70.0 \%$ of the patients, no change was observed in $25.0 \%$ and the condition decreased in $5.0 \%$. Multivariate regression analysis indicated that the body surface area and low hemoglobin levels were independent factors that caused changes in taste $(\mathrm{P}=0.003$ and $\mathrm{P}=0.021$, respectively) (50). Another multicenter, randomized, double-blind and placebo-controlled clinical study revealed that treatment with $68 \mathrm{mg}$ of polaprezinc daily for 12 weeks is also effective for patients with idiopathic taste disorders (51).

Treatment of chronic obstructive pulmonary disease. Kimura et al (52) used the human cancer lung epithelial cell line A549 to evaluate the effect of polaprezinc on cadmium-induced apoptosis. Polaprezinc was found to inhibit $\mathrm{CdCl}_{2}$-induced apoptosis of human lung epithelial cells. This inhibition suppressed the production of cadmium-dependent reactive oxygen species, significantly inhibiting the cadmium-induced apoptosis of lung epithelial cells. The study also demonstrated that the anti-oxidative effect of polaprezinc is mediated via the induction of metallothionein. Therefore, it is hypothesized that Polaprezinc might also be used to treat respiratory diseases such as chronic obstructive pulmonary disease.

\section{Safety}

In a previous study from our laboratory, specific adverse events that have been reported to be associated with high-dose zinc $(120 \mathrm{mg} / \mathrm{kg})$ daily were not observed (16); however, if required, zinc concentration may be kept under monitoring 
while treating patients with polaprezinc. Polaprezinc granules are a gastric mucosal protective drug, which can prevent acute injury of rat gastric and duodenal ulcer models and have a healing effect of chronic gastric ulcers in rat models. In the long-term toxicity test, rats present with salivation after oral administration of polaprezinc at a dose of $300 \mathrm{mg} / \mathrm{kg}$ or more for 13 weeks. Oral administration of polaprezinc at a dose of $600 \mathrm{mg} / \mathrm{kg}$ or more will cause a decrease in hemoglobin concentration, a decrease in hematocrit value and reticulocyte count, elevated glutamic-pyruvic transaminase, pathological examination of pancreatic fibrosis and erosions. A high dose of polaprezinc $(1,200 \mathrm{mg} / \mathrm{kg})$ causes side effects of sedation, erect hair, abdominal enlargement and diarrhea, in addition to a mortality rate of $40 \%$ in rats. The dead rats all presented with pancreatic fibrosis, thymus atrophy, adrenal cortex fatty degeneration and bone marrow suppression. The aforementioned adverse reactions caused by drugs can return to normal after drug withdrawal. In clinic, the main side effects of polaprezinc are nausea, vomiting, constipation or diarrhea. Some patients may have abnormal liver function, but these side effects are generally temporary and will eventually disappear. If the side effects are serious, other types of gastric drugs can be used for treatment. In addition, polaprezinc may interact with some other drugs. For example, when this product is taken simultaneously with penicillamine and levothyroxine sodium, it can form a chelate to reduce the absorption level of polaprezinc and reduce its curative effect. Therefore, taking these drugs together should be avoided. It is therefore crucial to inform the clinician of additional medications taken by the patient. The use of polaprezinc granules should be carried out under the guidance of the doctor (53).

The specific adherence of polaprezinc at the ulcer lesion is attributed to the formation of a new chemical bond between zinc and body components, such as albumin or other proteins to form mixed ligand complexes. L-carnosine still binds with $\mathrm{Zn}$ at this stage. These body components emerging from the ulcer site can bind strongly to zinc ions with functional groups such as sulfhydryl or imidazole. L-carnosine, which possesses wound healing properties owing to its free radical-scavenging properties, is released on the ulcer lesion via a complete ligand exchange reaction with a body component such as albumin or other proteins to form mixed ligand complexes capable of forming a complex of a larger stability constant than that of the complex formed from L-carnosine (21). Simultaneously, zinc with a protective effect on membranes is captured completely by the body component (such as albumin) and penetrates into the ulcer to ease inflammation (21). However, a typical composition of polaprezinc particles is $22 \%$ zinc and $78 \%$ L-carnosine (excipient is starch), which typically delivers 15-16 mg of zinc, which should not be a concern. In addition, polaprezinc has a long-established safety profile based on long-term use in humans and several preclinical and human clinical studies $(2,19,20,46)$ with no adverse events reported.

\section{Conclusions}

Apart from those in Japan, the number of patent applications for polaprezinc in China has increased. However, recent preparation routes and crystal-type inventions still show significant effects. Combined with other active ingredients, polaprezinc exerts evident synergistic effects and can therefore serves as a guide for the preparation of similar compositions with potentially improved synergy. Polaprezinc has multiple mechanisms of action, which vary from those of other mucosal protective agents. Comprehensive research on polaprezinc will help to expand the clinical application of polaprezinc.

\section{Acknowledgements}

Not applicable.

\section{Funding}

No funding was received.

\section{Availability of data and materials}

Not applicable.

\section{Authors' contributions}

ML and ZS designed the concept, wrote the manuscript, collected and sorted the literature and revised the article. HZ and ZL analyzed, interpreted the information and participated in the modification and revision. All authors have read and approved the final manuscript. Data sharing is not applicable.

\section{Ethics approval and consent to participate}

Not applicable.

\section{Patient consent for publication}

Not applicable.

\section{Competing interests}

The authors declare that they have no competing interests.

\section{References}

1. Seiki M, Ueki S, Tanaka Y, Soeda M, Hori Y, Aita H, Yoneta T, Morita H, Tagashira E and Okabe S: Studies on anti-ulcer effects of a new compound, zinc l-carnosine (z-103). Nihon Yakurigaku Zasshi 95: 257-269, 1990 (In Japanese).

2. Tan B, Luo HQ, Xu H, Lv NH, Shi RH, Luo HS, Li JS, Ren JL, Zou YY, Li YQ, et al: Polaprezinc combined with clarithromycin-based triple therapy for Helicobacter pylori-associated gastritis: A prospective, multicenter, randomized clinical trial. PLoS One 12: e0175625, 2017.

3. Wada I, Otaka M, Jin M, Odashima M, Komatsu K, Konishi N, Matsuhashi T, Horikawa Y, Ohba R, Itoh H and Watanabe S: Expression of HSP72 in the gastric mucosa is regulated by gastric acid in rats-Correlation of HSP72 expression with mucosal protection. Biochem Biophys Res Commun 349: 611-618, 2006.

4. Qin Y, Naito Y, Handa O, Hayashi N, Kuki A, Mizushima K, Omatsu T, Tanimura Y, Morita M, Adachi S, et al: Heat shock protein 70-dependent protective effect of polaprezinc on acetylsalicylic acid-induced apoptosis of rat intestinal epithelial cells. J Clin Biochem Nutr 49: 174-181, 2011.

5. Ohkawara T, Nishihira J, Nagashima R, Takeda H and Asaka M: Polaprezinc protects human colon cells from oxidative injury induced by hydrogen peroxide: Relevant to cytoprotective heat shock proteins. World J Gastroenterol 12: 6178-6181, 2006. 
6. Ueda K, Ueyama T, Oka M, Ito T, Tsuruo Y and Ichinose M: Polaprezinc (Zinc L-Carnosine) is a potent inducer of anti-oxidative stress enzyme, Heme Oxygenase (HO)-1-a new mechanism of gastric mucosal protection. Pharmacol Sci 110: 285-294, 2009.

7. Omatsu T, Naito Y, Handa O, Mizushima K, Hayashi N, Qin Y, Harusato A, Hirata I, Kishimoto E, Okada H, et al: Reactive oxygen species-quenching and anti-apoptotic effect of polaprezinc on indomethacin-induced small intestinal epithelial cell injury. J Gastroenterol 45: 692-702, 2010.

8. Shimada T, Watanabe N, Ohtsuka Y, Endoh M, Kojima K, Hiraishi $\mathrm{H}$ and Terano A: Polaprezinc Down-regulates proinflammatory cytokine-induced nuclear Factor-kappaB activation and Interleukin-8 expression in gastric epithelial cells. J Pharmacol Exp Ther 291: 345-352, 1999.

9. Handa O, Yoshida N, Tanaka Y, Ueda M and Yoshikawa T: Inhibitory effect of polaprezinc on the inflammatory response to Helicobacter pylori. Can J Gastroenterol 16: 785-789, 2002.

10. Ohata S, Moriyama C, Yamashita A, Nishida T, Kusumoto C, Mochida S, Minami Y, Nakada J, Shomori K, Inagaki Y, et al: Polaprezinc protects mice against endotoxin shock. J Clin Biochem Nutr 46: 234-243, 2010.

11. Watanabe S, Wang XE, Hirose M, Kivilioto T, Osada T, Miwa H, Oide H, Kitamura T, Yoneta T and Seto K: Insulin-like growth factor I Plays a role in gastric wound healing: Evidence using a zinc derivative, polaprezinc, and an in vitro rabbit wound repair model. Aliment Pharmacol Ther 12: 1131-1138, 1998.

12. Seto K, Yoneta T, Suda $\mathrm{H}$ and Tamaki H: Effect of polaprezinc (N-(3-aminopropionyl)-L-histidinato zinc), a novel antiulcer agent containing zinc, on cellular proliferation: Role of insulin-like growth factor I. Biochem Pharmacol 58: 245-250, 1999.

13. Jung DH, Park JC, Lee YC, Lee SK, Shin SK, Chung H, Park JJ, Kim JH, Youn YH and Park H: Comparison of the efficacy of Polaprezinc plus proton pump inhibitor and rebamipide plus proton pump inhibitor treatments for endoscopic submucosal dissection-induced ulcers. Clin Gastroenterol 55: 233-238, 2021.

14. Kono T, Asama T, Chisato N, Ebisawa Y, Okayama T, Imai K, Karasaki H, Furukawa H and Yoneda M: Polaprezinc prevents ongoing thioacetamide-induced liver fibrosis in rats. Life Sci 90 : $122-130,2012$

15. Katayama S, Nishizawa K, Hirano M, Yamamura $S$ and Momose Y: Effect of polaprezinc on healing of acetic acid-induced stomatitis in hamsters. J Pharm Pharm Sci 3: 114-117, 2000.

16. Liu Z, Xie W, Li M, Teng N, Liang X, Zhang Z, Yang Z and Wang X: Oral administration of polaprezinc attenuates fluorouracil induced intestinal mucositis in a mouse model. Basic Clin Pharmacol Toxicol 121: 480-486, 2017.

17. Liu ZY, Xie WB, Li MR, Teng N, Liang X and Zhang ZQ: Effects of polaprezinc on gastric mucosal damage and neurotransmitters in a rat model of chemotherapy-induced vomiting. Int Med Res 46: 2436-2444, 2018

18. Liu ZY, Xie WB, Li MR, Liu J, Liang X and Li T: Intrarectally administered polaprezinc attenuates the development of dextran sodium sulfate-induced ulcerative colitis in mice. Exp Ther Med 18: 4927-4934, 2019

19. Kashimura H, Suzuki K, Hassan M, Ikezawa K, Sawahata T, Watanabe T, Nakahara A, Mutoh H and Tanaka N: Polaprezinc, a mucosal protective agent, in combination with lansoprazole, amoxicillin and clarithromycin increases the cure rate of Helicobacter pylori infection. Aliment Pharmacol Ther 13: 483-487, 1999 .

20. Nishizawa T, Suzuki H, Kanai T and Yahagi N: Proton pump inhibitor alone vs proton pump inhibitor plus mucosal protective agents for endoscopic submucosal dissection-induced ulcer: A systematic review and meta-analysis. J Clin Biochem Nutr 56: 85-90, 2015.

21. Matsukura $\mathrm{T}$ and Tanaka $\mathrm{H}$ : Applicability of zinc complex of L-carnosine for medical use. Biochemistry (Mosc) 65: 817-823, 2000

22. Matsuda K, Mera Y, Wada H, Aruga H, Saik Y and Taniguchi Y: Repeated dose toxicity studies on catena-(S)-[mu-[N alpha-(3-aminopropionyl) histidinato(2-)-N1,N2,O:N tau]-zinc] in rats. Arzneimittelforschung 41: 1036, 1991.

23. Furuta S, Toyama S, Miwa M and Sano H: Disposition of polaprezinc (zinc L-carnosine complex) in rat gastrointestinal tract and effect of cimetidine on its adhesion to gastric tissues. J Pharm Pharmacol 47: 632-636, 1995.

24. Choi HS, Lim JY, Chun HJ, Lee M, Kim ES, Keum B, Seo YS, Jeen YT, Um SH, Lee HS, et al: The effect of polaprezinc on gastric mucosal protection in rats with ethanol induced gastric mucosal damage: Comparison study with rebamipide. Life Sci 93: 69-77, 2013.
25. Fujii Y, Matsura T, Kai M, Kawasaki H and Yamada K: Protection by polaprezinc, an anti-ulcer drug, against indomethacin-induced apoptosis in rat gastric mucosal cells. Jpn J Pharmacol 84: 63-70, 2000.

26. Yakoob J, Abbas Z, Jafri W, Usman MW and Awan S: Zinc chloride inhibits Helicobacter pylori growth and reduces expression of interleukin-1beta by gastric epithelial cells. Br J Biomed Sci 71: 43-45, 2014.

27. Ishihara R, Iishi $H$, Sakai N, Yano $H$, Uedo $N$, Narahara $H$, Iseki K, Mikuni T, Ishiguro S and Tatsuta M: Polaprezinc attenuates Helicobacter pylori-associated gastritis in Mongolian gerbils. Helicobacter 7: 384-389, 2010.

28. Naito Y, Yoshikawa T, Yagi N, Matsuyama K, Yoshida N, Seto K and Yoneta T: Effects of polaprezinc on lipid peroxidation, neutrophil accumulation, and TNF-alpha expression in rats with aspirin-induced gastric mucosal injury. Dig Dis Sci 46: 845-851, 2001.

29. Aita H, Yoneta T, Seto K, Morit H, Hori Y, Takemasa T, Chaki K, Yamada H, Seiki M and Tagashira E: Studies on the healing promoting action of Z-103 in chronic gastric ulcer models of rats. Nihon Yakurigaku Zasshi 99: 345-352, 1992 (In Japanese).

30. Odashima M, Otaka M, Jin M, Wada I, Horikawa Y, Matsuhashi T, Ohba R, Hatakeyama N, Oyake J and Watanabe S: Zinc L-carnosine protects colonic mucosal injury through induction of heat shock protein 72 and suppression of NF-kappaB activation. Life Sci 79: 2245-2250, 2006.

31. Ko JK and Leung CC: Ginger extract and polaprezinc exert gastroprotective actions by anti-oxidant and growth factor modulating effects in rats. J Gastroenterol Hepatol 25: 1861-1869, 2010.

32. Matsuoka S, Matsumura H, Nakamura H, Oshiro S, Arakawa Y, Hayashi J, Sekine N, Nirei K, Yamagami H, Ogawa M, et al: Zinc supplementation improves the outcome of chronic hepatitis C and liver cirrhosis. J Clin Biochem Nutr 45: 292-303, 2009.

33. Ye J, Zhang ZS, Zhu L, Lu FM, Li Y, Zhou JJ, Lu XL and Du Q Polaprezinc inhibits liver fibrosis and proliferation in hepatocellular carcinoma. Mol Med Rep 16: 5523-5528, 2017.

34. Matsumura H, Nirei $K$, Nakamura $H$, Arakawa $Y$, Higuchi T, Hayashi J, Yamagami H, Matsuoka S, Ogawa M, Nakajima N, et al: Zinc supplementation therapy improves the outcome of patients with chronic hepatitis C. J Clin Biochem Nutr 51: 178-184, 2012.

35. Amin M, Iqbal MS, Hughes RW, Khan SA, Reynolds PA, Enne VI, Sajjad-ur-Rahman and Mirza AS: Mechanochemical synthesis and in vitro anti-Helicobacter pylori and uresase inhibitory activities of novel zinc(II)-famotidine complex. J Enzyme Inhib Med Chem 25: 383-390, 2010.

36. Nishizawa $\mathrm{T}$ and Yahagi N: Endoscopic mucosal resection and endoscopic submucosal dissection: Technique and new directions. Curr Opin Gastroenterol 33: 315-319, 2017.

37. Inaba $\mathrm{T}$, Ishikawa $\mathrm{S}$, Toyokawa $\mathrm{T}$, Ishikawa $\mathrm{H}$, Miyahara $\mathrm{K}$, Wato M, Kawai K, Okada $\mathrm{H}$ and Yamamoto K: Basal protrusion of ulcers induced by endoscopic submucosal dissection (ESD) during treatment with proton pump inhibitors, and the suppressive effects of polaprezinc. Hepatogastroenterology 57: 678-684, 2010.

38. Watari I, Oka S, Tanaka S, Aoyama T, Imagawa H, Shishido T, Yoshida S and Chayama K: Effectiveness of polaprezinc for low-dose aspirin-induced small-bowel mucosal injuries as evaluated by capsule endoscopy: A pilot randomized controlled study. Gastroenterology 13: 108, 2013.

39. Doi H, Fujiwara M, Suzuki H, Niwa Y, Nakayama M, Shikata T, Odawara S, Takada Y, Kimura T, Kamikonya N and Hirota S: Polaprezinc reduces the severity of radiation-induced mucositis in head and neck cancer patients. Mol Clin Oncol 3: 381-386, 2015.

40. Hayashi H, Kobayashi R, Suzuki A, Ishihara M, Nakamura N, Kitagawa J, Kanemura N, Kasahara S, Kitaichi K, Hara T, et al: Polaprezinc prevents oral mucositis in patients treated with high-dose chemotherapy followed by hematopoietic stem cell transplantation. Anticancer Res 34: 7271-7277, 2014

41. Ishihama H, Sayo S, Yokoyama T, Ueno M, Ebihara N, Doi Y, Asano K, Kawamata H, Imai H and Ueki K: P1-032preventive and therapeutic effects of Polaprezinc suspension on oral mucosal injury. Ann Oncol 24: ix66-ix99, 2013.

42. Hayashi H, Kobayashi R, Suzuki A, Yamada Y, Ishida M, Shakui T, Kitagawa J, Hayashi H, Sugiyama T, Takeuchi H, et al: Preparation and clinical evaluation of a novel lozenge containing polaprezinc, a zinc-L-carnosine, for prevention of oral mucositis in patients with hematological cancer who received high-dose chemotherapy. Med Oncol 33: 91, 2016. 
43. Masayuki F, Norihiko K, Keita T, Miwa I, Masayuki I, Toshihiko I, Hiromi F, Chikaaki M and Norio N: Efficacy and safety of polaprezinc as a preventive drug for radiation-induced stomatitis. Nihon Igaku Hoshasen Gakkai Zasshi 62: 144-150, 2002 (In Japanese).

44. Doi H, Kamikonya N, Takada Y, Fujiwara M, Tsuboi K, Inoue H, Tanooka M, Nakamura T, Shikata T, Tsujimura T and Hirota S: Efficacy of Polaprezinc for acute radiation Proctitis in a rat model. Int J Radiat Oncol Biol Phys 80: 877-884, 2011.

45. Odawara S, Doi H, Shikata T, Kitajima K, Suzuki H, Niwa Y, Kosaka K, Tarutani K, Tsujimura T, Kamikonya N and Hirota S: Polaprezinc protects normal intestinal epithelium against exposure to ionizing radiation in mice. Mol Clin Oncol 5: 337-381, 2016.

46. Itagaki M, Saruta M, Saijo H, Mitobe J, Arihiro S, Matsuoka M, Kato T, Ikegami $\mathrm{M}$ and Tajiri H: Efficacy of zinc-carnosine chelate compound, Polaprezinc, enemas in patients with ulcerative colitis. Scand J Gastroenterol 49: 164-172, 2014.

47. Zhang Y, Okamura S, Kudo T, Masuo T and Mori M: Calcineurin inhibition by polaprezinc in rats with experimentally-induced colitis. Life Sci 88: 432-439, 2011.

48. Hewlings S and Kalman D: A review of Zinc-L-Carnosine and its positive effects on oral Mucositis, taste disorders, and gastrointestinal disorders. Nutrients 12: 665, 2020.
49. Takaoka T, Sarukura N, Ueda C, Kitamura Y, Kalubi B, Toda N, Abe K, Yamamoto S and Takeda N: Effects of zinc supplementation on serum zinc concentration and ratio of apo/holo-activities of angiotensin converting enzyme in patients with taste impairment. Auris Nasus Larynx 37: 190-194, 2010.

50. Mizukami Y, Sato J, Nihei S, Kashiwaba M, Kudo K, Okuyama H and Tamura K: The effectiveness of Polaprezinc preparation for taste alteration in cancer chemotherapy. Gan To Kagaku Ryoho 43: 979-983, 2016 (In Japanese).

51. Sakagami M, Ikeda M, Tomita H, Ikui A, Aiba T, Takeda N, Inokuchi A, Kurono Y, Nakashima M, Shibasaki Y and Yotsuya O: A zinc-containing compound, Polaprezinc, is effective for patients with taste disorders: Randomized, double-blind, placebo-controlled, multi-center study. Acta Otolaryngol 129: $1115-1120,2009$

52. Kimura K, Nakano Y, Sugizaki T, Shimoda M, Kobayashi N, Kawahara M and Tanaka KI: Protective effect of polaprezinc on cadmium-induced injury of lung epithelium. Metallomics 11: 1310-1320, 2019.

53. Japan standard commodity classification No. 872329: 11 2009. https://www.pmda.go.jp/PmdaSearch/iyakuDetail/ GeneralList/2329027. 